Ann. Zootech., I97I, 20 (3), 32I-338.

\title{
INFLUENCE DE LA VARIATION DE L'APPORT D'ALIMENT CONCENTRÉ, AVANT ET APRÈS L'CESTRUS INDUIT PAR TRAITEMENT HORMONAL, SUR LA FÉCONDITÉ DE LA BREBIS
}

\author{
R. GIROU*, M. THÉRIEZ, G. MOLÉNAT et D. AGUER \\ avec la collaboration technique de P. Dacheux et M. Dumont-Saint-Priest \\ Station de Recherches sur la Physiopathologie de la Nutrition* \\ Station de Recherches sur l'Elevage des Ruminants, \\ Centre de Recherches de Clermont-Ferrand, I. N.R. A., \\ 63 - Saint-Genès-Champanelle
}

\section{RÉSUMÉ}

La distribution d'aliment concentré pendant les 3 semaines qui précèdent l'insémination, et les 4 semaines qui la suivent, a modifié les résultats de reproduction de brebis à oestrus synchronisés à l'aide d'éponges vaginales imprégnées de progestagène et recevant de la $P$. M. S. G. (400 UI).

L'apport d'aliment concentré avant l'œstrus augmente les taux d'ovulation, de fertilité et de prolificité des brebis.

La distribution d'aliment concentré après l'insémination augmente la fertilité et la prolificité des brebis, à condition toutefois de limiter les quantités ingérées.

La suppression de l'aliment concentré, le lendemain de l'IA, a augmenté les pertes embryonnaires après le I $_{3}$ e jour, mais n'a pas eu d'effet sur les pertes précoces (mises en évidence par les retours en chaleurs avant le $2 \mathrm{I}$ e jour).

Nous n'avons pas observé de relation entre le gain de poids vif avant l'œstrus et les taux d'ovulation ou de prolificité. Ce sont les brebis les plus lourdes lors de l'insémination qui sont les plus prolifiques.

\section{INTRODUC'TION}

L'influence du niveau des apports alimentaires, lors de la lutte, sur la fécondité des brebis a fait l'objet de nombreux travaux. CoOP (I966 a), TASSEL (I967) et Hunter (I968) les ont présentés dans_des revues récentes que nous avons résumées dans le tableau $\mathrm{I}$.

L'élévation du niveau alimentaire ("flushing ») a uñeffet sur la fertilité mais

* Adresse actuelle : Maison de l'Élevage de la Mayenne, 53 - Laval. 
R. GIROU, M. THÉRIEZ, G. MOLÉNAT, D. AGUER

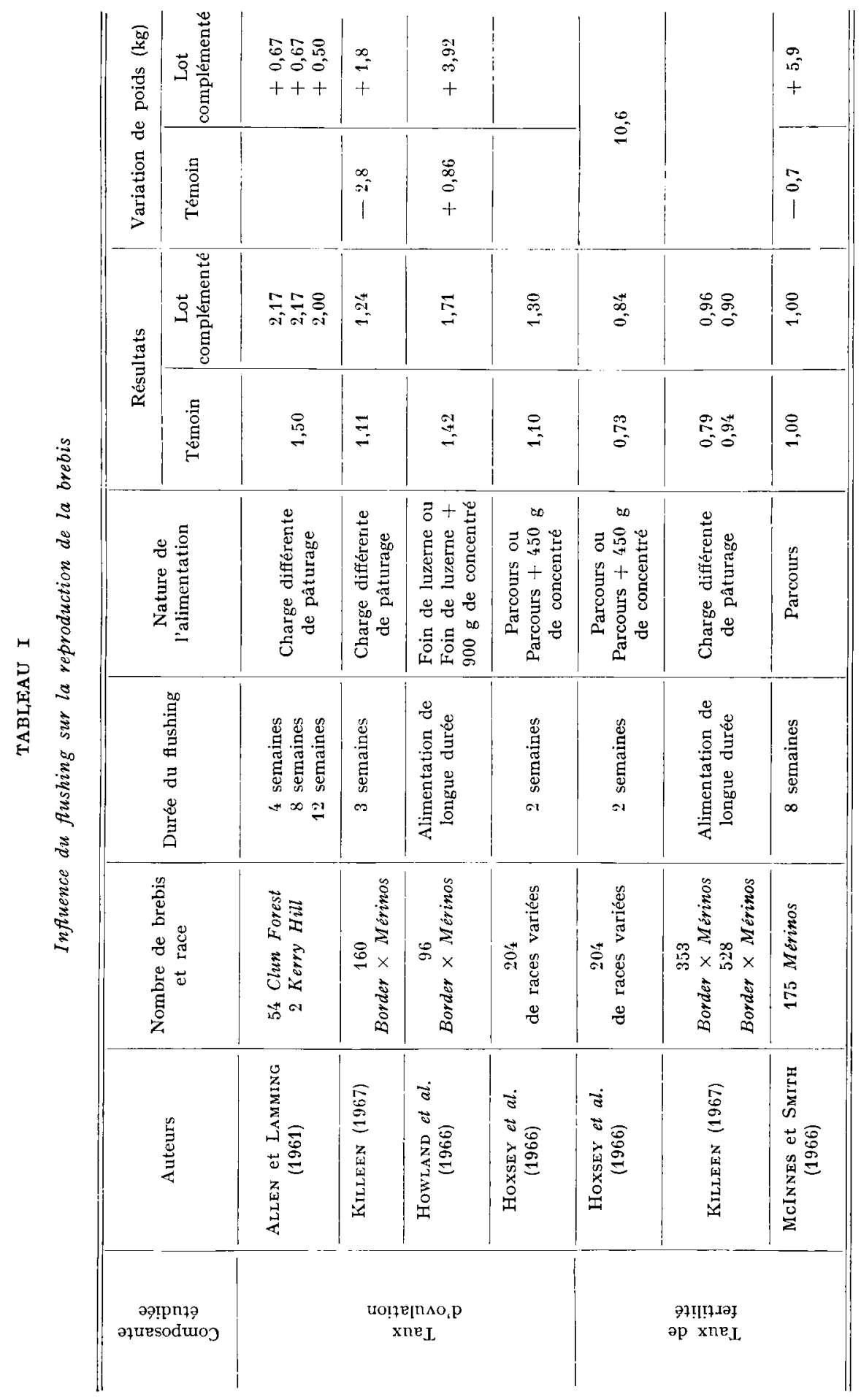


AIIMENTA'TION - FÉCONDITÉ DE LA BREBIS

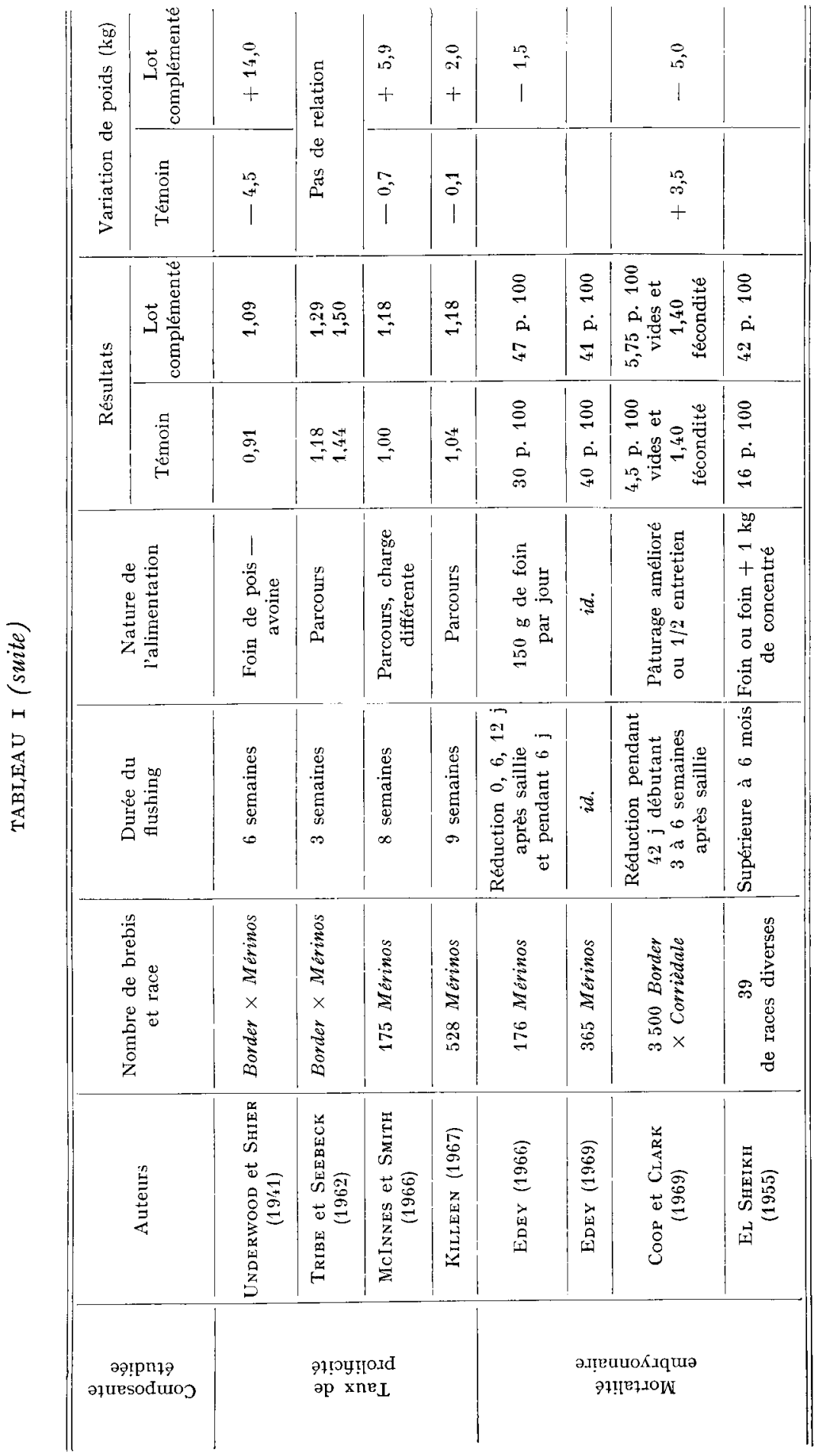


les résultats diffèrent suivant les auteurs. Selon WALIACE (I954), Hoxsey et al. (I960), GOETZEE et al. (1964) et COOP (I966 b), la proportion de brebis gestantes après la première saillie serait augmentée ; elle ne serait pas modifiée selon UNDERWOOD et SHIER (I94I) et KILLEEN (I967), et serait même diminuée selon WALLACE (r95I).

C'est essentiellement en améliorant la prolificité que l'effet favorable du flushing se manifeste sur la fécondité ( ${ }^{\mathbf{1}}$ ). La proportion de naissances gémellaires augmente par suite d'un accroissement du nombre d'ovulations multiples (ALLEN et LAMming, I96I ; BELLOWs et al., I963 $b$; KILLEEN, I967). Le gain de prolificité est cependant le plus souvent inférieur à l'augmentation du taux d'ovulation (KILILEN, I967; COOP, I966; HULET et al., Ig68) par suite d'une mortalité embryonnaire plus élevée chez les brebis à ovulations multiples: RobINSON (I957) ; QuinLIVAN et al. (I966) ; EDEY (I970).

L’influence de l'alimentation après la lutte a également été étudiée. Si le niveau alimentaire est nettement excédentaire (É, SHEIK et al., I955; FoOTE et al., I959) ou fortement déficitaire (BENNETT et al., I964; EDEY, I966 et I970) les pertes d'ovules et les pertes embryonnaires de début de gestation sont augmentées; si le niveau alimentaire est modéré, la fécondité ne serait pas modifiée (Coop, I966; BENNETT et al., I964; KILLEEN, Ig66).

Ces résultats ont été obtenus le plus souvent sur des brebis de race Mérinos ou croisées Mérinos, au pâturage et en lutte naturelle. La durée du flushing variait de 6 à 9 semaines, commençant 3 semaines avant l'introduction des béliers et s'achevant lorsque la majeure partie des brebis avait été saillie.

De telles conditions se rencontrent moins souvent en France où l'introduction des méthodes d'élevage utilisant les hormones exogènes pour le contrôle du cycle et l'accroissement du taux d'ovulation (Maulé́on, I966; Thimonien et al., i968) posent le problème de l'interférence de ces méthodes avec des techniques alimentaires (flushing) qui agissent par stimulation des sécrétions hormonales $\left(A_{L_{1} L_{i} N}\right.$ et LAMMing, I96I ; HowlaND et al., Ig66).

Ces diverses données nous ont amené à entreprendre l'étude des effets du régime alimentaire durant les 4 semaines qui précèdent et les 3 semaines qui suivent l'insémination sur les performances de reproduction d'un troupeau de brebis, à chaleurs synchronisées par un progestagène, recevant de la PMSG et dont le régime alimentaire correspond à celui couramment rencontré dans les bergeries en France.

\section{MATÉRIEL ET MÉTHODES}

Nous avons comparé au cours de deux expériences successives, l'une en septembre (expérience I), l'autre en décembre (expérience $\mathbf{I I}$ ), les taux d'ovulation, de retour en chaleurs avant (I968)

(1) Nous utiliserons les paramètres suivants définis selon la nomenclature proposée par IEESIGNES

$$
\begin{aligned}
& \text { - le taux de prolificité } \frac{\text { nombre d'agneaux nés }}{\text { nombre de brebis mettant bas }} \\
& \text { - le taux de fertilité } \frac{\text { nombre de brebis mettant bas }}{\text { nombre de brebis en expérience }} \\
& \text { - le taux de fécondité } \frac{\text { nombre d'agneaux nés }}{\text { nombre de brebis en expérience }}
\end{aligned}
$$


le $2 \mathrm{I}$ e jour et de prolificité de 329 brebis Limousines, âgées de $\mathrm{I} 8$ mois à 5 ans. Ces animaux, qui disposaient de foin à volonté, ont reçu différentes quantités d'aliment concentré pendant les 4 semaines précédant et les 3 semaines suivant l'insémination. Celle-ci a été faite lors de l'œestrus induit par traitement hormonal.

Nous avons réparti les brebis en 5 lots, dont deux, désignés sur la figure I sous les noms

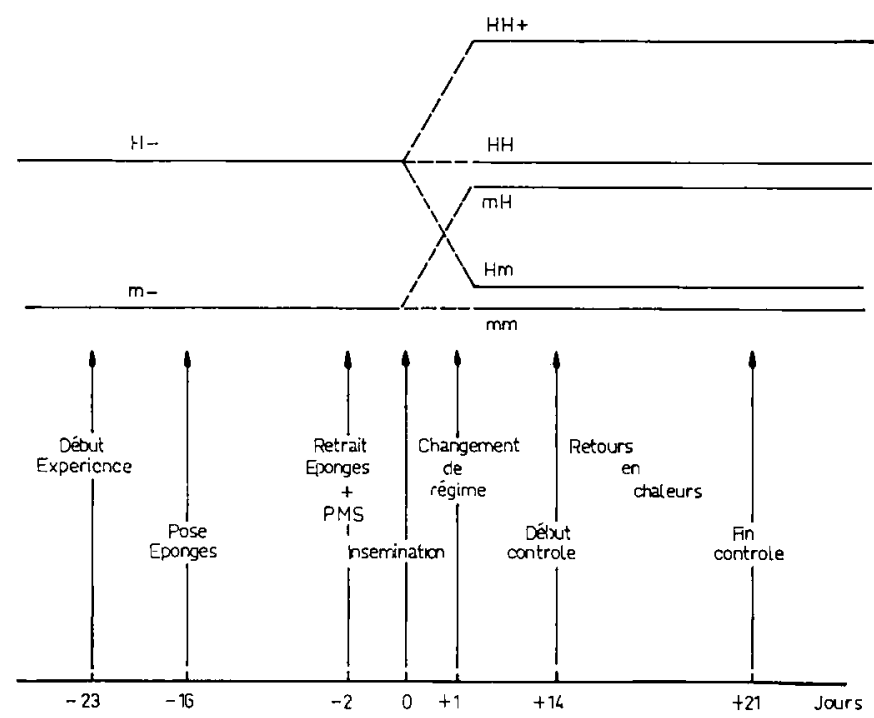

FIG. I. - Schéma et chronologie des expériences

de $m m$ et $\mathrm{HH}$, ont reçu un régime constant tout au long de l'expérience, alors que le régime des 3 autres lots variait le lendemain de l'insémination.

Les animaux du lot $\mathrm{mm}$, dont le niveau alimentaire était modéré, constituaient les témoins, tandis que ceux du lot HH étaient destinés à mettre en évidence l'effet de l'apport d'aliment concentré sur les différents critères de reproduction utilisés et présentés ci-dessus.

\section{TABLEAU 2}

Régime alimentaive des diffévents lots

\begin{tabular}{|c|c|c|}
\hline Régimes & Expérience I & Expérience II \\
\hline $\begin{array}{l}m \\
\mathrm{H} \\
\mathrm{H}^{+}\end{array}$ & $\begin{array}{l}1,45 \mathrm{~kg} \text { de foin } \\
1,35 \mathrm{~kg} \text { de foin }+300 \mathrm{~g} \text { concentré } \\
1,35 \mathrm{~kg} \text { de foin }+500 \mathrm{~g} \text { concentré }\end{array}$ & $\begin{array}{l}\text { Foin * }+200 \mathrm{~g} \text { concentré } \\
\text { Foin * }+500 \mathrm{~g} \text { concentré } \\
\text { Foin * +700g concentré }\end{array}$ \\
\hline
\end{tabular}

* La consommation moyenne de foin pour l'ensemble du troupeau était de l'ordre de $1,6 \mathrm{~kg}$ par brebis/jour.

Le lendemain de l'insémination, l'apport de concentré a été augmenté pour 2 lots (lots $\mathrm{mH}$ et $\mathrm{HH}^{+}$) alors qu'il a été réduit pour le $3^{\mathrm{e}}(\operatorname{lot} \mathrm{Hm})$. Ces lots devaient permettre la mise en évidence des effets de la variation du niveau alimentaire sur les pertes embryonnaires en début de gestation (fig. I : schéma expérimental). 
Les lots constitués en début d'expérience étaient homogènes quant à l'âge et au poids des brebis. Celles-ci pesaient $48,5 \mathrm{~kg}$ en moyenne lors de l'expérience I $(48,25$ pour le lot témoin, 48,75 pour le lot flushing) et $53,0 \mathrm{~kg}$ lors de l'expérience II (soit respectivement $52,9 \mathrm{et} 53,0 \mathrm{~kg}$ ).

Des brebis ont dû être éliminées en cours d'expérience, soit par suite de maladie, soit parce qu'elles avaient perdu leur éponge vaginale avant la date de retrait. Ces éliminations ont entraîné des écarts entre les poids moyens des lots, écarts qui atteignent $2 \mathrm{~kg}$ entre le lot $\mathrm{mm}$ et le lot $\mathrm{HH}$, lors de l'expérience I ; au contraire, les lots de l'expérience II sont plus voisins.

Le foin de pré, de qualité moyenne, provenait d'origines diverses. L'aliment concentré était un mélange qui contenait 47,5 p. Ioo de granulés de luzerne déshydratée ( 8 p. Ioo de matière azotée totale), 47,5 p. Ioo de céréales (orge en automne, maîs en hiver) et $5 \mathrm{p}$. Ioo de minéraux (tabl. 2).

\section{Conduite de l'expérience}

Expérience I : les brebis qui étaient auparavant au pâturage, ont changé progressivement de régime en une semaine puis sont rentrées en bergerie où l'on a constitué autant de lots qu'il y avait de régimes différents.

Expérience II : les brebis des différents lots constituaient un seul troupeau qui était parqué dans les bois, en plein air. Les animaux avaient donc accès aux mêmes rateliers, ils n'étaient séparés et répartis dans les groupes expérimentaux respectifs que pour la distribution du concentré.

Au cours de l'expérience I, nous avons mesuré chaque jour les quantités de foin offert et refusé tandis que dans l'expérience II, nous avons pesé globalement les remorques de foin distribué. Les quantités d'aliment concentré consommées ont été pesées tous les jours.

\section{Technique de reproduction}

Les chaleurs des brebis ont été synchronisées à l'aide d'éponges vaginales imprégnées de $30 \mathrm{mg}$ d'acétate de fluorogestone (Robinson, I967; Thimonier et al., i968 a et b). Lors du retrait des éponges, I 4 jours après leur pose, on a injecté 4 oo UI de P. M. S. G. aux brebis. Nous les avons inséminées 48 et $60 \mathrm{~h}$ plus tard, avec du sperme frais, sans détection préalable des chaleurs. Dans l'expérience I, les brebis qui étaient encore en chaleurs $7^{2} \mathrm{~h}$ après le retrait des éponges ont été de nouveau inséminées.

Le sperme, prélevé sur 6 béliers Limousins et 3 béliers Romanov, était dilué selon la technique et avec le dilueur proposés par la Station de Physiologie de la Reproduction de l'I. N. R. A. (ColAs, I969). Dans l'expérience I, la concentration du sperme dilué était d'environ $300 \times$ Io $^{\mathbf{6}}$ spermatozoïdes par dose de $0,5 \mathrm{cc}$. Dans l'expérience II, nous avons utilisé pour chaque brebis, $0,5 \mathrm{cc}$ de sperme dilué à raison de I volume de sperme pour 4 volumes de dilueur. Les béliers ont été répartis uniformément entre les différents lots expérimentaux.

\section{Mesures}

Les brebis ont été pesées en début et en fin d'expérience ainsi que lors de l'œstrus. Les pesées avaient lieu deux jours consécutifs à la même heure.

Entre le $5^{\mathrm{e}}$ et le $8 \mathrm{e}$ jour après l'insémination, nous avons déterminé par endoscopie (THımoNiER et Mauleon, I969) le taux d'ovulation de I 44 brebis (65 en automne, 79 en hiver) soit 40 p. roo de l'ensemble du troupeau en expérience. L'examen des ovaires nous a permis de déterminer le nombre de corps jaunes présents. Dans 4 cas, le nombre d'agneaux nés a été supérieur au nombre de corps jaunes observés sur les ovaires, et dans ces 4 cas, les agneaux étaient du même sexe. Compte tenu des observations de Rowson et Moor (I964) qui fixent à I p. Ioo environ le taux de jumeaux vrais, nous avons estimé que ces agneaux étaient des jumeaux vrais et nous avons assimilé le nombre d'ovules pondus au nombre de corps jaunes observés.

Nous avons détecté les retours en chaleurs entre le $\mathrm{I}_{4}{ }^{\mathrm{e}}$ et $\mathrm{le} 2 \mathrm{I}$ jour après l'insémination à l'aide de béliers vasectomisés (MAUleoN et DaUzIER, I965). Les brebis, ainsi détectécs, que nous désignerons sous le terme de "brebis vides au $2 \mathrm{I}$ e jour ", sont revenues en chaleurs soit parce qu'elles n'avaient pas été fécondées (pas d'ovulation, ovulation silencieuse, non. fécondation), soit parce qu'elles avaient perdu précocement tous leurs embryons avant le $\mathrm{I} 3$ e jour de gestation, date de transformation du corps jaune cyclique en corps jaune gestatif (EDEY, r969). Nous les distinguons des "brebis gestantes au $2 \mathrm{I}^{\mathrm{e}}$ jour " dont certaines n'ont pas agnelé car elles ont subi des pertes embryonnaires totales après $1 \mathrm{e} I 3^{\mathrm{e}}$ jour. 


\section{RÉSULTATS}

\section{Poids vif et état nutritionnel}

Les brebis qui avaient disposé d'un pâturage abondant dans l'intervalle entre les deux essais, étaient plus lourdes au début de la seconde expérience $(53 \mathrm{~kg}$ contre $48 \mathrm{~kg}$ ). Cette différence de poids, qui traduit une différence d'état, entraîne, au moins partiellement, un accroissement du taux d'ovulation lors du second essai.

Quel que soit leur régime antérieur, tous les animaux ont gagné du poids avant la lutte : $3 \mathrm{~kg}$ lors du rer essai ; $0,3 \mathrm{~kg}$ lors du second. Seules les brebis qui ne rece-

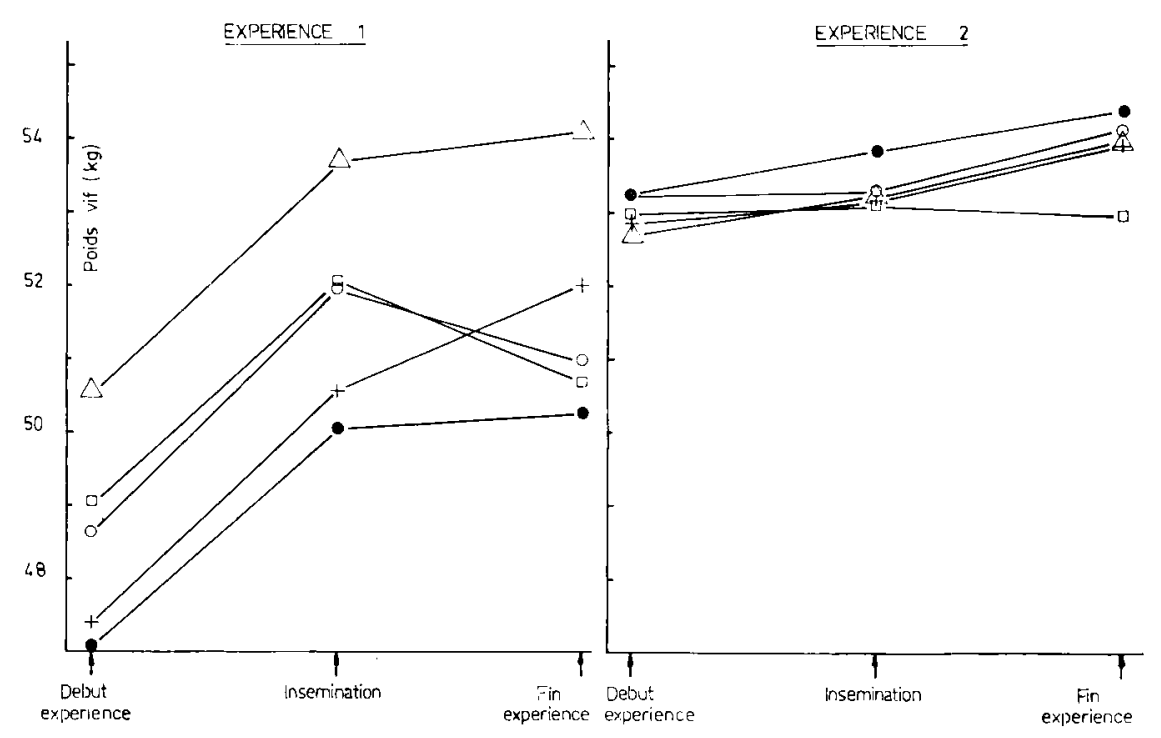

Fig. 2. - Evolution du poids vif des brebis

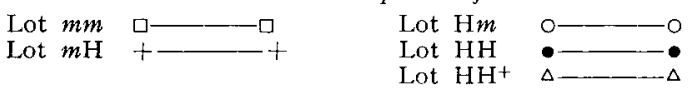

vaient que du foin (lot $\mathrm{H} m$ et $m m$ du premier essai) après l'œstrus ont maigri. Cette chute de poids peut expliquer l'importance des pertes embryonnaires dans ces deux lots (72 et 44 p. IOO).

Outre les pesées, nous avons estimé l'état nutritionnel des brebis au cours des différentes phases de l'expérience par le dosage du glucose et des acides gras libres du sang. Le prélèvement avait lieu le matin, avant distribution du concentré, sur 24 animaux (I2 par régime). Nous avons déterminé la teneur en acides gras libres par la méthode de Dole et Meynerth (I966) et celle du glucose par la glucose oxydase (HILL, et KESSLER, I96I).

Les teneurs en glucose sanguin sont plus élevées chez les animaux complémentés cependant les écarts sont faibles et nous n'avons obtenu de différences significatives que dans un seul cas, à la fin de l'expérience I (tabl. 3).

Inversement, les animaux complémentés ont des teneurs en acides gras libres 
R. GIROU, M. THÉRIEZ, G. MOLÉNAT, D. AGUER

sanguins moins élevées que les témoins. Les écarts sont plus importants que pour le glucose et la différence est significative à la fin de chacun des 2 essais (tabl. 3 ).

TABI,EAU 3

Teneurs en acides gras libres et en glucose sanguin

\begin{tabular}{|c|c|c|c|c|c|c|}
\hline \multirow{2}{*}{ Date } & \multicolumn{3}{|c|}{ Glucose $(\mathrm{mg} / 100 \mathrm{ml})$} & \multicolumn{3}{|c|}{ Acides gras libres (méq./ml) } \\
\hline & Témoin & Complémenté & & Témoin & Complémenté & \\
\hline $\begin{array}{c}16 / 9 \\
\text { Insémination } \\
7 / 10 \\
\text { Fin de l'expérience }\end{array}$ & 56,4 & $\begin{array}{l}67,6 \\
63,2\end{array}$ & $\begin{array}{c}\text { NS } \\
\mathrm{P}<0,05\end{array}$ & $\begin{array}{l}341,0 \\
401,9\end{array}$ & 318,0 & $\begin{array}{c}\text { NS } \\
\mathrm{P}<0,05\end{array}$ \\
\hline $\begin{array}{c}16 / 1 \\
\text { Insémination } \\
16 / 1 \\
\text { Fin de l'expćrience }\end{array}$ & 35,3 & 33,4 & $\begin{array}{l}\text { NS } \\
\text { NS }\end{array}$ & $\begin{array}{l}192,0 \\
116,1\end{array}$ & 198,0 & $\begin{array}{c}\text { NS } \\
P<0,05\end{array}$ \\
\hline
\end{tabular}

Taux de non retour en chaleurs au $21^{\mathrm{e}}$ jour et taux de maintien de la gestation

On constate, dans l'expérience $I$, que le taux de non retour en chaleurs au $2 \mathrm{I}$ e jour est amélioré notablement par le flushing préostral $\left(0,68\right.$ dans le lot $m-\left({ }^{1}\right)$ contre 0,85 dans le lot $\mathrm{H}-; p<0,02$ ) (tabl. 4). Celui-ci n'a pas d'action sur le maintien de la gestation après le $\mathrm{I} 3^{\mathrm{e}}$ jour. A l'inverse, le flushing postœstral, quoiqu'il manifeste une légère tendance à augmenter le taux de non retour en chaleurs au $2 \mathrm{I}^{\mathrm{e}}$ jour $\left(0,73\right.$ dans le lot $-m$ contre 0,78 dans les lots $-\mathrm{H}$ et $\left.\mathrm{HH}^{+}\right)$exerce une action favorable essentiellement sur le maintien de la gestation $(0,78$ dans le lot-- $m$ contre 0,88 dans le lot $-\mathrm{H}$ et $\left.\mathrm{HH}^{+} ; p<0,05\right)$.

Dans l'expérience II, aucune différence significative n'apparaît entre les différents lots. Toutefois, on note une tendance à l'abaissement du taux de non retour dans le lot $\mathrm{HH}^{+}(0,76$ contre $0,84,0,82,0,85,0,83)$.

\section{Taux de fertilité}

Dans l'expérience I, le taux de fertilité varie fortement selon le niveau alimentaire avant et après l'insémination (tabl. 4). Les différences entre les lots $\mathrm{HH}^{+}$, $\mathrm{HH}$ et le lot $\mathrm{mm}(0,73$ et 0,74 contre $0,5 \mathrm{I})$ sont hautement significatives et celles entre les lots $\mathrm{H}$ - et $m-(0,7 \mathrm{I}$ contre 0,57$)$ sont proches de la signification $(p<0,06)$.

Le taux de fertilité est nettement augmenté par un niveau alimentaire élevé sur toute la durée de l'expérience, il a tendance à être plus haut à la fois sous l'action

(1) $m$ - désigne le lot de brebis ayant reçu le régime $m$ avant l'IA sans tenir compte du régime après l'IA. Il regroupe les lots $m m$ et $m \mathrm{H}$.

- $m$ désigne le lot de brebis ayant reçu le régime $m$ après l'IA sans tenir compte du régime avant l'IA. Il regroupe les lots $\mathrm{mm}$ et $\mathrm{Hm}$ 
d'un flushing préœestral $(0,7 \mathrm{I}$ contre 0,57$)$ et sous 1'action d'un flushing postcstral $(0,69$ contre 0,57$)$. Le premier intervenant essentiellement sur la réussite de l'insémination et le deuxième sur le maintien de la gestation, on comprend que leurs effets apparaissent comme additifs. Les lots $\mathrm{H} m$ et $m \mathrm{H}(0,62$ et 0,67$)$ se situent à un niveau intermédiaire entre le lot $m m$ et les lots $\mathrm{HH}$ et $\mathrm{HH}^{+}$.

TABLEAU 4

Taux de non retour en chaleur au $21^{\mathrm{e}}$ jour.

Taux de maintien de la gestation au-delà du $21^{\mathrm{e}}$ jour et taux de fertilité

\begin{tabular}{|c|c|c|c|c|c|c|c|c|c|}
\hline & \multicolumn{5}{|c|}{ Lots } & \multicolumn{4}{|c|}{ Régimes } \\
\hline & $m m$ & $m \mathrm{H}$ & $\mathrm{H} m$ & $\mathrm{HH}$ & $\mathrm{HH}+$ & $m-$ & $\mathrm{H}-$ & $-m$ & $-\mathrm{H}$ \\
\hline \multicolumn{10}{|l|}{ Expérience I } \\
\hline Nombre de brebis inséminées. & 41 & 40 & 27 & 27 & 26 & 81 & 80 & 68 & 67 \\
\hline Nombre de brebis agnelant . & 21 & 25 & 18 & 20 & 19 & 46 & 57 & 39 & 45 \\
\hline Taux de non retour ....... & 0,66 & 0,70 & 0,85 & 0,85 & 0,85 & 0,68 & 0,85 & 0,73 & 0,78 \\
\hline Taux de maintien ........ & 0,78 & 0,89 & 0,78 & 0,87 & 0,86 & 0,84 & 0,84 & 0,78 & 0,88 \\
\hline Taux de fertilité $\ldots \ldots \ldots$ & 0,51 & 0,62 & 0,67 & 0,74 & 0,73 & 0,57 & 0,71 & 0,57 & 0,67 \\
\hline \multicolumn{10}{|l|}{ Expérience II } \\
\hline Nombre de brebis inséminées. & 43 & 40 & 27 & 29 & 29 & 83 & 85 & 70 & 69 \\
\hline Nombre de brebis agnelant.. & 35 & 22 & 21 & 22 & 21 & 67 & 64 & 56 & 54 \\
\hline Taux de non retour $\ldots . .$. & 0,84 & 0,82 & 0,85 & 0,83 & 0,76 & 0,83 & 0,81 & 0,84 & 0,82 \\
\hline Taux de maintien ........ & 0,97 & 0,97 & 0,91 & 0,92 & 0,95 & 0,97 & 0,93 & 0,95 & 0,95 \\
\hline Taux de fertilité ......... & 0,81 & 0,80 & 0,78 & 0,76 & 0,72 & 0,81 & 0,75 & 0,80 & 0,78 \\
\hline \multicolumn{10}{|l|}{ Total } \\
\hline Nombre de brebis inséminées. & 84 & 80 & 54 & 56 & 55 & 164 & 165 & 138 & 136 \\
\hline Nombre de brebis agnelant. . & 56 & 57 & 39 & 42 & 40 & 113 & 121 & 95 & 99 \\
\hline Taux de non retour ....... & 0,75 & 0,76 & 0,85 & 0,84 & 0,84 & 0,75 & 0,84 & 0,78 & 0,79 \\
\hline Taux de maintien $\ldots \ldots \ldots$ & 0,89 & 0,93 & 0,85 & 0,89 & 0,91 & 0,91 & 0,88 & 0,87 & 0,92 \\
\hline Taux de fertilité.... & 0,67 & 0,71 & 0,72 & 0,75 & 0,73 & 0,69 & 0,73 & 0,69 & 0,73 \\
\hline
\end{tabular}

Dans l'expérience II, le taux de fertilité ne présente que peu de variations avec le niveau alimentaire. Toutefois, il a tendance à diminuer quand le niveau alimentaire augmente : $0,8 \mathrm{I}$ dans le lot $m m, 0,80$ dans le lot $\mathrm{Hm}, 0,78$ dans le lot $m \mathrm{H}$, 0,76 dans le lot $\mathrm{HH}$ et 0,72 dans le lot $\mathrm{HH}^{+}$.

Aucune liaison n'est observée entre le taux de fertilité et le poids vif, sur l'ensemble des deux essais.

\section{Taux d'ovulation}

Pour l'ensemble des deux expériences, l'apport de concentré avant l'œstrus a augmenté le taux d'ovulation de 0,20 points ( $I, 96$ dans le lot $\mathrm{H}$ - contre I,76 dans le lot $m-; p<0,05)$. Ce gain a été du même ordre de grandeur $(+0,25$ et $+0,18$ ovules/brebis) au cours du premier essai, en début de saison sexueile, sur 
des brebis en état moyen que lors du second essai, en milieu de saison sexuelle, avec des animaux plus lourds, et en meilleur état, et dont le taux d'ovulation était significativement plus élevé (tab1. 5).

\section{TABLEAU 5}

Taux d'ovulation

\begin{tabular}{|c|c|c|c|c|c|c|}
\hline \multirow[b]{2}{*}{ Lots } & \multicolumn{2}{|c|}{ Expérience I } & \multicolumn{2}{|c|}{ Expérience II } & \multicolumn{2}{|c|}{ Total } \\
\hline & $\begin{array}{c}\text { Nombre } \\
\text { d'animaux }\end{array}$ & $\begin{array}{c}\text { Taux } \\
\text { d'ovulation }\end{array}$ & $\begin{array}{c}\text { Nombre } \\
\text { d'animaux }\end{array}$ & $\begin{array}{c}\text { Taux } \\
\text { d'ovulation }\end{array}$ & $\begin{array}{l}\text { Nombre } \\
\text { d'animaux }\end{array}$ & $\begin{array}{c}\text { Taux } \\
\text { d'ovulation }\end{array}$ \\
\hline $\begin{array}{l}m- \\
\mathrm{H}-\end{array}$ & $\begin{array}{l}31 \\
34\end{array}$ & $\begin{array}{l}1,48 a \\
1,73 b\end{array}$ & $\begin{array}{l}39 \\
40\end{array}$ & $\begin{array}{l}1,97 c \\
2,15 d\end{array}$ & $\begin{array}{l}70 \\
74\end{array}$ & $\begin{array}{l}1,76 e \\
1,96 f\end{array}$ \\
\hline
\end{tabular}

Interprétation statistique : $a-b \quad \mathrm{P}<0,05$

$\begin{array}{ll}a-c & \mathrm{P}<0,01 \\ b-d & \mathrm{P}<0,05 \\ e-f & \mathrm{P}<0,05\end{array}$

$\begin{array}{ll}e-f & \mathrm{P}<0,05 \\ c-d & \mathrm{P}<0,05\end{array}$

TABLEAU 6

Relation entre le poids vif lors de l'cestrus et les taux d'ovulation et de prolificité

\begin{tabular}{|c|c|c|c|c|c|c|c|}
\hline \multirow{2}{*}{ Lots } & \multirow{2}{*}{$\begin{array}{l}\text { Nbre d'ovules } \\
\text { par brebis }\end{array}$} & \multicolumn{2}{|c|}{ Expérience I } & \multicolumn{2}{|c|}{ Expérience II } & \multicolumn{2}{|c|}{ Total } \\
\hline & & Effectif & Poids & Effectif & Poids & Effectif & Poids \\
\hline$m-$ & $2 \underset{1}{2}$ ou plus & $\begin{array}{l}17 \\
12\end{array}$ & $\begin{array}{l}52,5 * \\
45,6 \text { * }\end{array}$ & $\begin{array}{l}27 \\
12\end{array}$ & $\begin{array}{l}52,1 \\
52,0\end{array}$ & $\begin{array}{l}44 \\
24\end{array}$ & $\begin{array}{l}52,2 \\
48,8\end{array}$ \\
\hline $\mathrm{H}-$ & $\underset{1}{2 \text { ou plus }}$ & $\begin{array}{l}24 \\
10\end{array}$ & $\begin{array}{l}53,3 \\
50,0\end{array}$ & $\begin{array}{l}30 \\
10\end{array}$ & $\begin{array}{l}54,4 \\
51,4\end{array}$ & $\begin{array}{l}54 \\
20\end{array}$ & $\begin{array}{l}53,9 \\
50,7\end{array}$ \\
\hline & $\begin{array}{c}\text { Nbre d'agneaux } \\
\text { par brebis }\end{array}$ & & & & & & \\
\hline$m-$ & $\begin{array}{c}2 \text { ou plus } \\
1 \\
0\end{array}$ & $\begin{array}{l}20 \\
26 \\
35\end{array}$ & $\begin{array}{l}54,2 \\
50,5 \\
50,0\end{array}$ & $\begin{array}{l}34 \\
33 \\
16\end{array}$ & $\begin{array}{l}55,0 \\
51,6 \\
52,0\end{array}$ & $\begin{array}{l}54 \\
59 \\
51\end{array}$ & $\begin{array}{l}54,7 \\
51,1 \\
51,0\end{array}$ \\
\hline $\mathrm{H}-$ & $\begin{array}{c}2 \text { ou plus } \\
1 \\
0\end{array}$ & $\begin{array}{l}30 \\
27 \\
23\end{array}$ & $\begin{array}{l}52,4 \\
50,9 \\
49,2\end{array}$ & $\begin{array}{l}38 \\
26 \\
21\end{array}$ & $\begin{array}{l}52,5 \\
52,6 \\
56,6\end{array}$ & $\begin{array}{l}68 \\
53 \\
4 ;\end{array}$ & $\begin{array}{l}52,4 \\
51,7 \\
52,9\end{array}$ \\
\hline
\end{tabular}

$* \mathrm{P}<0,05$. 
Les brebis à ovulations multiples sont plus lourdes que celles qui ne présentent qu'une ovulation simple. La différence de poids entre ces deux catégories d'animaux est significative pour l'ensemble des deux essais $(53,2 \mathrm{~kg}$ contre $49,7 \mathrm{~kg} ; p<0,05)$; elle est hautement significative dans l'expérience I $(53,0 \mathrm{~kg}$ contre $47,6 \mathrm{~kg} ; p<0,0 \mathrm{I})$ mais ne l'est pas dans l'expérience II $(53,4 \mathrm{~kg}$ contre $5 \mathrm{I}, 3 \mathrm{~kg})$. Par contre, le poids moyen des brebis à ovulations multiples est le même $(53,0$ et $53,3 \mathrm{~kg})$ au cours des deux essais malgré des différences d'état d'entretien, de niveau alimentaire et de date. (Tabl. 6).

\section{Taux de prolificité}

Pour l'ensemble des deux expériences, c'est le lot $\mathrm{HH}$ qui a eu le meilleur taux de prolificité (tabl. 7).

I,e flushing préœstral seul, améliore le taux de prolificité de o,Io points, c'est-àdire de la moilié du gain obtenu sur le taux d'ovulation.

Le flushing postœstral a un effet favorable dans le premier essai, mais non dans le second.

Lors du premier essai, les brebis qui n'ont mis bas qu'un agneau, étaient, au moment de l'œstrus, significativement moins lourdes que les bessonières $(50,6 \mathrm{~kg}$ contre $53,2 \mathrm{~kg} ; p<0,0 \mathrm{I})$. L'écart n'est pas significatif lors du second (52, I contre $54,6)$.

Au cours de chacune des deux expériences, le poids moyen des brebis ayant eu des naissances multiples est plus faible dans les lots $\mathrm{H}-$ que dans les lots $m$-. Le flushing préostral a donc permis d'obtenir une proportion plus importante de gestations multiples chez les brebis légères. Si nous n'avions pas observé un tel effet sur le taux d'ovulation, ceci est peut-être dî au fait que nous n'avons examiné qu'un nombre restreint d'animaux (I44), sur une population de 329 .

\section{Taux de fécondité}

Le taux de fécondité étant le produit de taux de prolificité par le taux de fertilité, les constatations que nous allons effectuer découlent de celles déjà citées.

Dans l'expérience I, les différences entre les lots $\mathrm{HH}, \mathrm{HH}^{+}$et $\mathrm{mm}$ sont significatives $(p<0,03)$ et les différences entre les lots $m$ - et $\mathrm{H}$ - ainsi qu'entre les lots $-m$ et $\mathrm{HH}, \mathrm{HH}^{+}$sont proches de la signification $(p<0,08)$. Le niveau alimentaire élevé sur toute la durée de l'expérience permet donc d'avoir le meilleur taux de fécondité ( $\mathrm{I}, \mathrm{I} 5$ et $\mathrm{I}, \mathrm{I} 8$ contre $0,7 \mathrm{I}$ ). Ce dernier est augmenté à la fois par le flushing préœstral ( $\mathrm{I}, 09$ contre 0,82 ) et par le flushing postcestral ( $\mathrm{I}, 09$ contre o,79) (iabl. 7)

Dans l'expérience II, aucune différence significative n'apparaît ; toutefois, le taux de fécondité a tendance à être abaissé dans les lots $m \mathrm{H}(\mathrm{I}, 22)$ et surtout $\mathrm{HH}^{+}$ $(\mathrm{I}, \mathrm{IO})$, lots où le niveau alimentaire est augmenté après insémination.

\section{Pertes d'ovules et d'embryons}

I a proportion d'ovules non représentés par des naissances, varie de 22 à $72 \mathrm{p}$. Ioo selon les différents lots de brebis examinées à l'endoscope. Ces variations sont ré- 
R. GIROU, M. THÉRIEZ, G. MOLÉNAT, D. AGUER

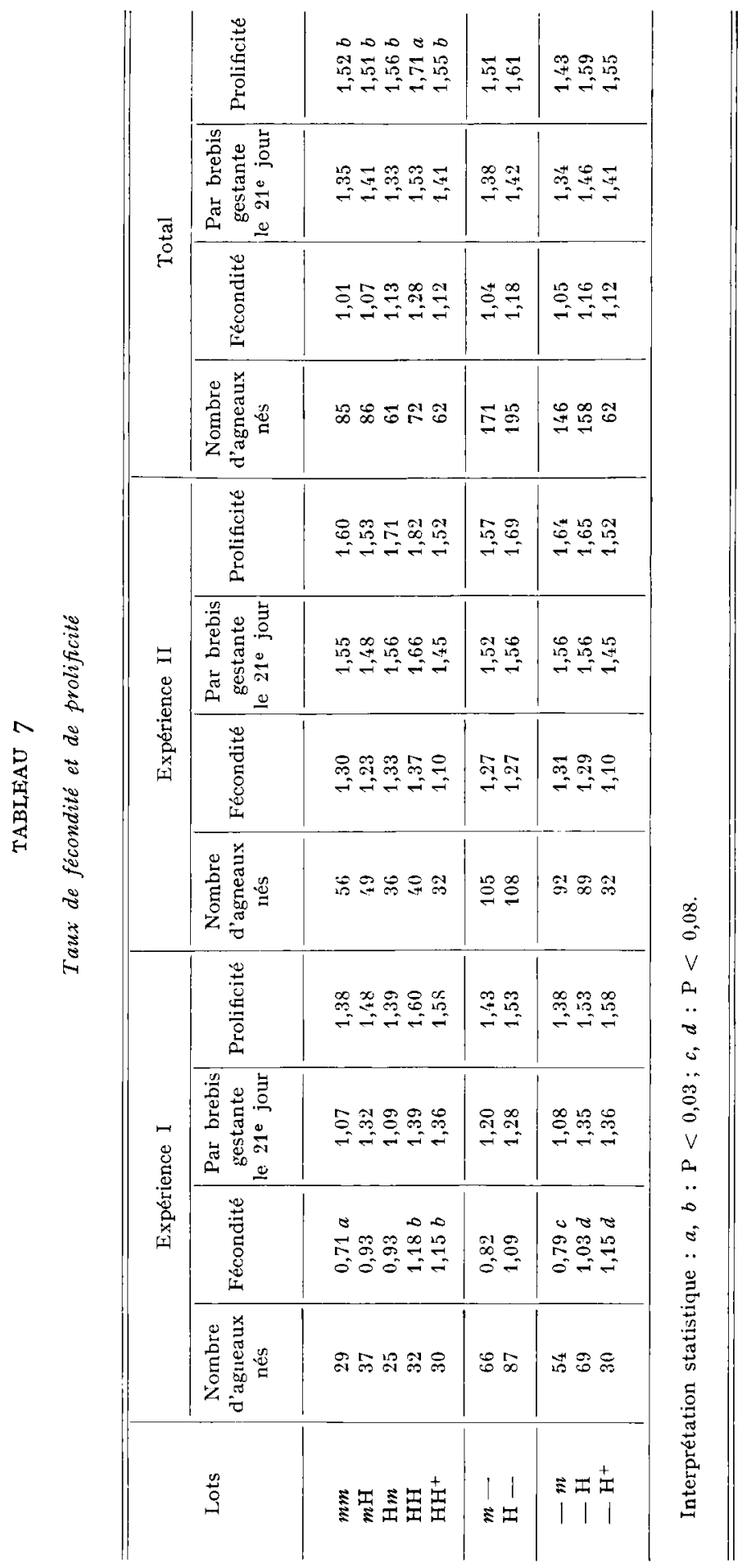


duites si l'on regroupe les lots correspondants des deux essais ou si l'on considère l'ensemble de la population de brebis utilisées au cours de l'expérience ( 40 à 48 p. Ioo).

Un apport régulier et important de concentré, avant et après l'œstrus, permet de réduire les pertes d'ovules. Celles-ci sont égales dans les lots $\mathrm{mm}$ et $\mathrm{HH}$ (33 p. IOo), bien que les taux d'ovulation de ces deux lots diffèrent significativement.

Une distribution de concentré avant 1'œstrus $(\mathrm{H}-)$ permet de réduire le nombre de brebis revenant en chaleurs avant le $2 \mathrm{I}^{\mathrm{e}}$ jour, donc les pertes d'ovules. De même,

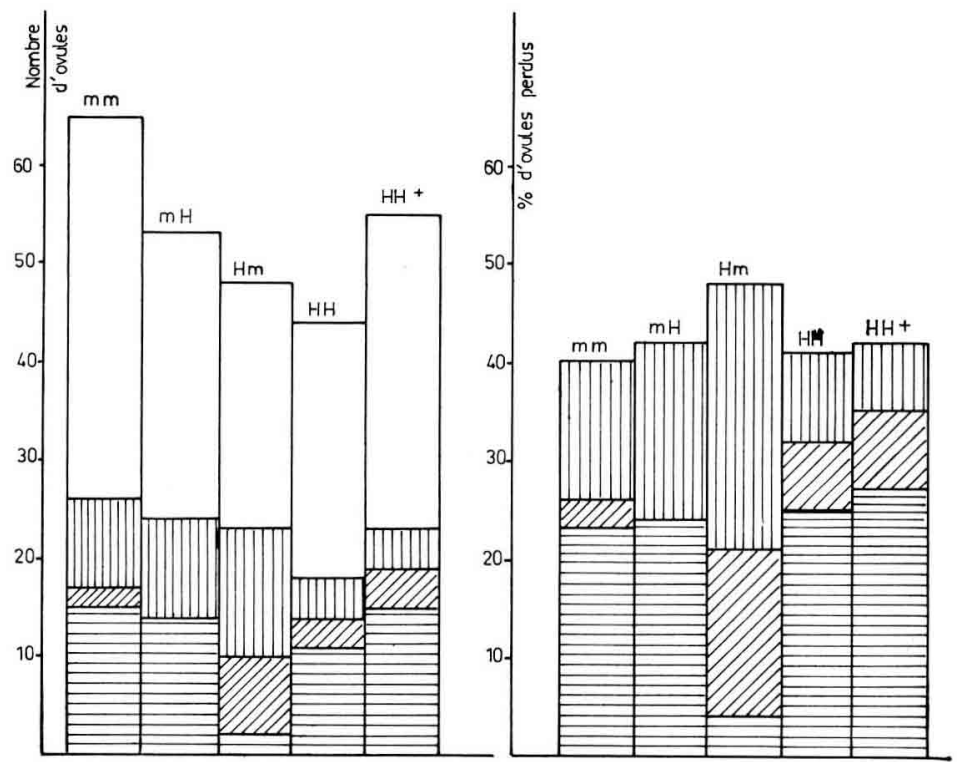

FIG. 3. - Pertes embryonnaires des brebis ayant subi l'endoscopie

IIIII Pertes partielles : perte d'une partie des ovules ou embryons

Pertes totales avant le $\mathrm{I} 3^{\mathrm{e}}$ jour : perte de tous les ovules ou embryons

QDZ Pertes totales après le $13^{\mathbf{e}}$ jour

la distribution de concentré après l'œstrus $(-\mathrm{H})$ permet d'accroître la proportion de brebis qui poursuivent leur gestation au-delà du $2 I^{\mathrm{e}}$ jour et donc de réduire les pertes embryonnaires.

Inversement, la réduction ou l'accroissement important et brutal de l'apport de concentré le lendemain de l'œstrus augmente les pertes embryonnaires (lots $\mathrm{H} m$ et $\mathrm{HH}^{+}$) (fig. 3).

\section{DISCUSSION ET CONCLUSION}

Les deux expériences dont nous avons rapporté les résultats, ont un caractère essentiellement zootechnique. Nous avons voulu vérifier s'il était possible de généraliser, dans des conditions différentes d'élevage, les relations entre niveau alimen- 
taire en période de lutte et fécondité de la brebis établies par UNDERwood et SHIER (I94I) ; AlLen et LAMming (I96I); CoOP (I962, I964, I966) et KILLEEN (I967).

Nous avons utilisé un grand nombre de brebis, à deux époques de la saison sexuelle et dans un état corporel différent. La synchronisation des chaleurs par traitement hormonal, nous a permis de dissocier les effets du niveau alimentaire et de ses variations lors de l'œstrus sur les critères de reproduction.

I,es résultats confirment les données antérieures obtenues sur des troupeaux extensifs. Nous pouvons les résumer comme suit :

- Le flushing précstral a amélioré de 0,2 point le taux d'ovulation des brebis, ce qui correspond aux observations de ALLEN et LAMMING (I96I) et BELLOws et al. (I963), et de KILLEEN (r967) (tabl. I) ;

- il a accru la fertilité chez les animaux qui ne recevaient que du foin auparavant; il l'a au contraire diminuée si le régime initial comportait déjà des aliments concentrés. Son action se traduit par l'amélioration du taux de non retour en chaleurs avant le $2 \mathrm{x}^{\mathrm{e}}$ jour dans le premier cas (HoXSEY et al., I960; WALLACE, I95I), par l'accroissement des pertes embryonnaires précoces dans le second (EL SHEIK et al., I955; FoO'TE et al., I959; CURL et ALBIN, I970). Ces résultats sont analogues à ceux que Franzos (I968), Tyrrei et al. (I968,) Girou et Brochar'T (I970) ont obtenus sur des vaches laitières;

- la variation de régime le lendemain de l'insémination a un effet important et variable sur la mortalité embryonnaire. Une diminution de l'apport de concentré accroîtrait les pertes (E,DEY, I966) alors qu'une amélioration du régime permettrait de les réduire (Hoxsey et al., I960). Dans ce cas, la mortalité embryonnaire reste cependant supérieure à celle que l'on observe sur les brebis à régime constant.

Par contre, certains résultats diffèrent; nous les analyserons plus en détail.

\section{I. - Infuence des hormones exogènes}

L'influence du niveau alimentaire au moment de la lutte sur la fécondité des brebis n'a été que rarement étudiée sur des animaux à œestrus synchronisés à l'aide de progestatifs et recevant une injection de P. M. S. G.

Lorsque la dose d'hormone injectée est élevée (600 à I ooo UI) les taux d'ovulation sont les mêmes en dépit d'une alimentation différente dans les essais de WALLACE (I954) et ceux de ALLEN et LAMMING (I96I). Au contraire, avec les doses plus faibles, que nous avons utilisées (40O UI), les brebis qui ont reçu une complémentation avant la lutte ont eu un taux d'ovulation supérieur à celui des animaux témoins.

L'injection de P. M. S. G. provoque une sécrétion accrue d'œstrogènes. Dans le premier cas, ces hormones, sécrétées en quantité plus importante en réponse à une forte dose de P. M. S. G., ont agi par un mécanisme de rétroaction sur l'hypophyse, masquant ainsi les effets éventuels de 1'alimentation. Dans nos essais, la faible dose de P. M. S. G. utilisée, a accru la croissance folliculaire des ovaires, sans provoquer de phénomènes sensibles de rétroaction des oestrogènes sur l'hypophyse. Celle-ci a pu réagir aux différences de niveau alimentaire.

Le flushing que l'on considère souvent comme une technique d'élevage destinée à corriger les insuffisances de l'alimentation demeure donc applicable à des troupeaux menés de manière intensive dont l'alimentation est régulière et abondante. L'emploi 
des hormones exogènes à faibles doses pour synchroniser les chaleurs des brebis et à favoriser la ponte ovulaire, ne condamne donc pas cette pratique.

\section{2. - Mode d'action du fushing}

La distribution de concentré avant la lutte a permis d'accroître la fécondité et 1a prolificité des brebis, résultats dus en partie à une augmentation du taux d'ovulation. Si ce dernier a été amélioré de 0,2 point dans chacune des deux expériences, le gain n'a pas été obtenu de la même manière dans chaque cas :

- dans l'expérience I, le flushing a augmenté le pourcentage de brebis à ovulations doubles ( 55 p. Ioo pour $m-$; 7I p. IOO pour $\mathrm{H}-$ ). I1 n'a pas eu d'effet par contre sur le nombre de brebis à ovulations supérieures à 2 ;

- dans l'expérience II au contraire, le pourcentage de brebis à ovulations doubles a été légèrement accru par le flushing $(+6 \mathrm{p}$. I00) mais c'est surtout le pourcentage de brebis à ovulations supérieures à 2 qui a augmenté $(27,5 \mathrm{p}$. Ioo dans le lot $\mathrm{H}$-, I 9,9 p. Ioo dans le lot $m-$ ).

Le flushing a donc agi non seulement sur les brebis légères dont il a amélioré 1a prolificité, mais il a également augmenté les taux d'ovulation et de prolificité de celles qui étaient les plus lourdes. L'effet "dynamique " du flushing se manifesterait donc sur toutes les brebis, quel que soit leur état. Cette observation rejoint celle de Coop (I966) qui estime que la pratique consistant en un amaigrissement des animaux suivi d'une phase de gain de poids avant la lutte, est néfaste et donne de moins bons résultats que le maintien des brebis à un poids constant.

Nous avons observé des effets du flushing sur le taux d'ovulation et la prolificité des brebis mais nous n'avons pas pu mettre en évidence de relation entre ces résultats et le gain de poids ou les teneurs en glucose et en acides gras libres du sang. Tribe et SeEbeck (I962), CoOp (I966) ou Killieen (I967) ont constaté également qu'il pouvait y avoir un effet de flushing sans liaison avec le gain de poids des brebis. Par contre, Howl,AND et al. (I966) ont montré qu'il existait une relation très étroite entre les teneurs en glucose et acides gras libres du sang et le taux d'ovulation. Cependant, l'écart entre les régimes distribués étaient beaucoup plus fort que dans nos essais (900 $\mathrm{g}$ de céréales en supplément pour HowLAND, $300 \mathrm{~g}$ dans notre cas) et la durée de l'expérience était également beaucoup plus longue; nous n'avons d'ailleurs obtenu d'écart significatif dans les teneurs en glucose et acides gras libres sanguins, qu'après 6 semaines de régime différentiel.

Ces variations de la concentration en métabolites sanguins, qui agissent par stimulation de 1'hypothalamus et de l'hypophyse et par là sur l'activité ovarienne (Wallace, I954; Allen et Lamming, I96r ; Howland et al., I966), pourraient également modifier la sensibilité de l'ovaire aux hormones exogènes utilisées (HAMMOND, I952). Nos résultats ne permettent d'infirmer aucune de ces deux hypothèses. $\mathrm{Si}$ nous avons un effet du flushing malgré l'apport de 400 UI de P.M. S. G., cela peut être dû soit à une libération plus importante d'hormones hypophysaires chez les brebis complémentées, soit à une sensibilité accrue de l'ovaire à l'action hormonale de la P. M. S. G. 


\section{3. - Relation entre les variations du niveau alimentaire et la mortalité embryonnaire}

EDEY, qui a étudié au cours de 4 années successives l'influence d'une diminution très brutale du niveau alimentaire entre le $7^{\mathrm{e}}$ et le $20^{\mathrm{e}}$ jour après la saillie (ration réduite à $I^{\circ} \mathrm{g}$ de foin par jour), n'a obtenu de résultats significatifs que dans deux essais. A l'opposé, Er, SHEIK et al. (I955) ou Foote et al. (I959) ont observé une réduction de la fécondité chez des brebis qui recevaient une alimentation très riche en concentré depuis plusieurs mois.

Dans les deux cas, ces résultats ont été obtenus avec des régimes extrêmes qui ne correspondaient pas aux conditions normales d'élevage, ce qui n'est pas le cas pour les essais que nous rapportons où la variation de l'apport de concentré ne dépasse pas $300 \mathrm{~g}$. Par contre, nous avons modifié le régime des brebis à un moment bien déterminé $\mathrm{d} u$ cycle, à savoir le lendemain de l'insémination. La différence entre nos résultats et ceux de Coop et CLARK (I969) ou ceux de EDEY (I966 et I970) est certainement due, au moins en partie, à la date de réduction du niveau alimentaire.

L'accroissement de la mortalité embryonnaire qui en résulte entraîne des retours en chaleurs tardifs (au-delà $d u 2 I^{e}$ jour) et des pertes embryonnaires partielles. Ceci traduit une action lente de la variation de régime alimentaire, comme nous l'avions observé lors du dosage de métabolites sanguins.

\section{Reçu pour publication en mars 1971.}

\section{SUMMARY}

\section{INFLUENCE ON FERTILITY IN THE EWE OF VARYING THE PROPORTION}

\section{OF CONCENTRATES BEFORE AND AFTER OESTRUS INDUCED BY HORMONAL TREATMENT}

The rates of ovulation, return on heat before the 2 ist day and prolificity of 329 Limousin ewes aged between 2 and 5 years were compared during two successive experiments (september and december). Oestrus was synchronized by means of vaginal sponges, and when these were, removed, the sheep were injected with PMSG.

The ewes, divided into 5 dietary groups, were all given pasture hay ad libitum. During the three weeks preceding oestrus, half of the flock was flushed ( $300 \mathrm{~g}$ concentrates as a supplement). The day after insemination, the proportion of concentrates was either maintained, reduced or increased in 3 of the groups (fig. I and table 2 ).

I. - All the sheep gained weight during the 3 weeks before œestrus (fig. 2). The gain was the same, independent of the proportion of concentrates given.

2. - The sheep that were flushed had a significantly higher ovulation rate than the controls (table 5). (table 4 ).

3. - Pre-œstral flushing improved the fertility of the sheep during the first experiment

4. - The proportion of sheep in which gestation continued beyond the 2 Ist day after insemination was lower in the groups that only received hay (table 4 ).

5. - The fertility varied between $5 \mathrm{I} \mathrm{p.} \mathrm{Ioo,} \mathrm{in} \mathrm{the} \mathrm{control} \mathrm{group} \mathrm{that} \mathrm{only} \mathrm{had} \mathrm{hay} \mathrm{both}$ before and after insemination, to $80 \mathrm{p}$. I00, in the groups that also received concentrates during this period. The sheep that received concentrates for only part of the experiment (either before or after œstrus), showed an intermediate fertility : 64 p. Ioo (table 4 ). 
6. - The prolificity of the sheep was improved by the distribution of concentrates before cestrus (table 7). It was also improved by the addition of a small amount of concentrates to the prolificity (700 g).

7. - The highest fertility was obtained by distributing 300 to $500 \mathrm{~g}$ of concentrates from the $3^{\text {rd }}$ week before $\infty$ strus until the $4^{\text {th }}$ week afterwards. It was reduced either by stopping the supply of concentrates or by giving an excess (7oo g/sheep/day) (table 7 ).

8. - Sheep having had two ovules weighed $53 \mathrm{~kg}$ on the average, independent of the proportion of concentrates given or the date of œstrus (table 6). The sheep which produced two lambs were lighter in weight during cestrus in the groups that had been flushed. We have not observed any relation between the live weight or the weight gain and fertility or embryonic mortality.

9. - The same embryonic mortality rate occurred both in the sheep that received concen* trates during the whole experiment, and those that did not receive any concentrates, even though the rate of ovulation was significantly higher in the former (fig. 3).

\section{RÉFÉRENCES BIBLIOGRAPHIQUES}

Allen D. M., Lamming G. E., I96r. Nutrition and reproduction in the ewe. J. Agric, Sci, 56,69-79.

Bellows R. A., Pope A. L., Meyer R. K., Chapman A. B., Casida L. E., ig63a. Physiological mechanism in nutritionally induced differences in ovarian activity of mature ewes. J. Anim. Sci., 22, $93^{-100 .}$

Beliows R. A., Pope A. L., Chapman A. B., Casida L. E., I963 b. Effect of level and sequence of feeding and breed on ovulation rate, embryo survival and fetal growth in the mature ewe. J. Anim. Sci., 22, IOI-108.

Bellows R. A., Pope A. L., Chapman A. B., Casida L. E., r96r. Attempt to flush mature ewes with a sodium propionate drench and pelleted rations. J. Anim. Sci., 20, 965 (Abstr.) cité par BELLows et al., I963 $a$.

Bellows R. A., Meyer R. K., Hoekstra W. G., Casida L. E., ig66. Pituitary and ovarian activity in rats on two levels of dietary energy. J. Anim. Sci., 25, $38 \mathrm{r}-3^{85}$.

Bennett D., Axelsen A., Chapman H. M., i964. The effect of nutritional restriction during early pregnancy on number of lambs born. Proc. Aust. Soc. Anim. Prod., 5, 7o-72.

Colas G., 1969. Techniques nouvelles de reproduction des ovins : développements récents de l'insémination artificielle ovine. Journées $A, F$. Z, I6-17 décembre 1969, 4, 1-Io.

Coop I. E., Ig66a. The response of ewes to flushing. Wrld. Rev. Anim. Prod., 4, 69-73.

Coop I. E., I966 b. Effect of flushing on reproductive performance of ewes. J. Agric. Sci., 67, 305-323.

Coop I. E., CLARK V. R., I969. Influence of nutritional level in early pregnancy of ewe. J.Agric. Sci., 73, 387-393.

Curd S. E., Albin R. C., 1970. Ration effect on fertility, survival and growth of sheep. J. Anim. Sci., 30, 3I7, Abst.

Desvignes A., I968. Proposition de définition de critères zootechniques et économiques en matière d'élevage ovin. F. E. Z. Commission de Prod. Ovine et caprine. Dublin, Mai r966.

Dole V.P., Meinertz H., I960. Microdetermination of long chain fatty acids in plasma and tissues. J. Biol. Chem., 235, 2595-2599.

EDEY T. N., I966. Nutritional stress and pre implantation embryonic mortality in Merino sheep. J. A gric. Sci., 67, 287-293.

Edey T. N., 1968. Body weight and ovulation rate in sheep. Proc. Aust. Soc. Anim. Prod., 7, 188-19I.

Edey T. N., 1969. Prenatal mortality in sheep : a review. Anim. Breed. Abst., 37, I73-I83.

EDEY T. N., I97o. Nutritional stress and pre implantation mortality in Merino sheep. 1964-7. General discussion and conclusions. J. Agric. Sci., 74, I99-204.

El Sheik A. S., Hulet C. V., Pope A. L., Casida L. E., i955. The effect of level of feeding on the reproductive capacity of the ewe. J. Anim. Sci., 14, 919-929.

Franzos G., 1968. The relationship between the milk fat percentage and fertility in dairy herd. Refuah. Vet., 25, 28-32.

Girou R., Brochart M., I97o. Niveau énergétique, protéique et fécondité des vaches laitières. Influence d'une supplémentation alimentaire post-oestrale. Ann. Zootech., 19, 67-73.

Goetzee C, G., Vermeulen E. J., Dyanson J. E., ig64. Fing S. Afr., 40, 52. Cité par Tassel (ig67).

HAmmond J., I952. Fertility Chap. 2I, in Marshall's physiology of reproduction. Vol. 2, Ed. by A.S. Parkes. London, New York and Toronto : Longmans, Green and Co 3rd ed. 648-740. Cité par Hunter, I 968 .

Annales de Zootechnie. - 1971 . 
Hill J. B., Kessi.er J, Ig6r. An automated determination of glucose utilizing a glucose oxydase peroxidase system. J. Lab. and Clin. Med., 57, 970 .

Howland B. F., Kirpatrik R. L., Pope A. L., Casida L. F., ig66. Pituitary and ovarian function in ewes fed on two nutritional levels. J. Anim. Sci, 25, 716-721.

Hoxsey Vic, Hoversland A. S., Blackmore D. W., Van Horn J. L., 196o. Effect of pre breeding and post breeding treatments on reproductive phenomena of range ewes, J.Anim. Sci., 19, 959 (Abst.).

Hulet C. V., PRICE D. A., Foote W. C., 1968. Fffects of variation in light, month of year and nutrient intake on reproductive phenomena in ewes during the breeding season. J. Anim. Sci., 27, $684-690$.

Hunter G. L., I 968 . Increasing the frequency of pregnancy in sheep. I. Some factors affecting rebreeding during the post partum period. Anim. Breed. Abst., 36, 347-364.

KILLEEN A. D., I 967 . The effects of bodyweight and level of nutrition before during and after joiring on ewe fertility. Aust. J. Exp. Agric. Anim. Husb., 25, 126-1 36.

Mauléon P., Dauzier L., I965. Variation de la durée de l'anostrus chez la brebis de race Ile-deFrance. Ann. Biol. anim. Bioch. Biophys., 5, 132-143.

Maulíon P., 1966. Use of gonadotropins for raising, the fertility of domestic mammals. Proc. 5th W. Cong. on Fert. and Stcr. Stockholm. Exerpta Medica International Cong, series no 138, 35-42.

Quinlivan T. D., Martin C. A., Tayior W. B., Cairney I. M., ig66. Estimates of pre an perinatal mortality in those ewes that conceived to one service. J. Reprod. Fert., 11, 379-390.

Robinson T. J., 1957. Pregnancy. Progress in the physiology of farm animals p. 793. Ed. J. Hammond, Butterworths, London.

RoBinson T. J., I967. The control of the ovarian cycle in the sheep. 258 pp. Ed. T. J. Robinson. Sydney University press.

Rowson L. E., Moor R. M., 1964. Occurence of development of identical twins in sheep. Nature, 201, $521-522$.

TASSEL R., I967. The effects of diet on reproduction in pigs, sheep and cattle. III. Plane of nutrition in sheep. Br. Vet. J., 123, $257-264$.

Thimonier J., Mauléon P., Cognie Y., Ortavant R., ig68 a. Déclenchement de l'œstrus et obtention de la gestation chez des agnelles à l'aide d'éponges vaginales imprégnćes d'acétate de fluorogestone. Ann. Zootech., 17, 275-288.

Thimonier J., Maulíon P., Cognie Y., Ortavant R., ig68 b. Déclenchement de l'œestrus et obtention de la gestation pendant l'anostrus post-partum chè la brebis à l'aide d'éponges vaginales imprégnées d'acétate de fluorogestone. Ann. Zootech., 17, 257-273.

Thimonier J., MaulEon P., i 969 . Variations saisonnières du comportement d'œestrus et des activités ovariennes et hypophysaires chez la brebis. Ann. Biol. anim. Bioch. Biophys., 9, 233-25I.

Tribe D. E., SEebrck R. M., I962. Effect of live weight change on the lambing performance of ewes. J. Agric. Sci, 59, IO5-III.

Tyreil H. F., Trimberger G. W., Morrow D. A., Merell W. G., Reid J. T., Loosli J. K., 1968. Liberal grain feeding of dairy cows. Proc. Cornell Conference in feed Manuf. Oct 29-3I, 9.-Ioz.

UNDERwood E. J., SHIER F. L., I94I. Studies in sheep husbandry in western Australia. I. The effect of nutritional "flushing" upon fertility. J. Agric. West. Aust., 18 (2nd series), I3-2I.

Wallace L. R., I95I. Flushing of ewes, New Zel. J. Agric., 83, 377.

WALlace L. R., I 954 . Studies in the augmentation of fertility of Rommey ewes pregnant-mare serum. J. Agric. Sci., 45, 60-79. 